\title{
A Matched Field Processing Based on Compressed Sensing
}

\author{
Yingchun Chen ${ }^{\mathrm{a},{ }^{*}}$, Yali Jiang ${ }^{\mathrm{b}}$, Biao Wang ${ }^{\mathrm{c}}$ \\ Department of Electronic Information Engineering, Jiangsu University of Science and Technology, \\ Zhenjiang, Jiangsu, China \\ a2216314310@qq.com, b494951443@qq.com, c237873905@qq.com
}

\begin{abstract}
Keywords: Underwater Acoustic Localization; Compressed Sensing; Matched Field Processing; Sparse Reconstruction
\end{abstract}

\begin{abstract}
The traditional MFP (matched field processing, MFP) methods of underwater acoustic target often have poor estimation performance or get inaccurate estimation result on the constrain of spatial sparse observation. Considering the problem, this paper proposed a new high-accuracy MFP estimation algorithm of underwater acoustic target based on compressed sensing by analyzing the space sparsity of underwater target location. The algorithm established the spatial sparse description model of underwater target, and compressed sensing the underwater target in spatial domain, then used the joint sparse reconstruction algorithm to achieve the MFP estimation of underwater acoustic target. The simulation results show that the method can increase the DOA estimation accuracy of underwater acoustic target at less array elements and less snapshots.
\end{abstract}

\section{Introduction}

Underwater acoustic source localization is divided into active and passive sonar positioning. Compared with active sonar positioning, passive sonar localization has the characteristics of high security, high concealment and it can detect a further distance. MFP (matched field processing, MFP) plays an important role in passive target detection who combines analysis of characteristics of array signal processing and acoustic propagation in the ocean [1].In recent years, matched field processing technology has attracted much attention in the field of underwater target detection and passive acoustic localization. The Bartlett processor is proposed by Baggeroer and it has a good tolerance of the changes. Minimum variance distortionless response (MVDR) which can restrain the disturbance of the side lobe and the noise signal proposed by Capon is the most typical adaptive matched field processing. During the follow-up study, it is found that the side lobe is high when Bartlett processor is used to deal with the underwater acoustic localization and it leads to a larger fuzzy region. MVDR processors require precise parameters, a large number of samplings and high requirements for hardware and software [2].

In order to overcome the problems above, this paper proposed a new high-accuracy MFP estimation algorithm of underwater acoustic target based on spatial compressive sampling by analyzing the space sparsity of underwater target location. This method can realize the high precision positioning in the situation of few array elements.

\section{Method Principle}

Compressed sensing [3] is a kind of compressive sampling method which is different from traditional signal sampling. Compressed sensing theory said that when the signal is sparse or compressible, signal sampling reconstruction can be transformed into a problem of solving the uncertain linear observation equation. The steps to get the sparse signal through compressed sensing are sparse representation of signal, encoding measurement and reconstruction algorithm. According to compressed sensing, the signal is compressed while sampling. After the sparse representation of the signal, the received signal can be expressed as:

$$
\boldsymbol{Y}(l)=\boldsymbol{G X}(l)+N(l)
$$


$\mathrm{G}$ is a $\mathrm{M} * \mathrm{Q}$ dimensional projection matrix. $\mathrm{Y}(\mathrm{L})$ is a $\mathrm{M} \times 1$ dimensional compressive sampling data. $\mathrm{X}(\mathrm{L})$ is a $\mathrm{Q} \times 1$ dimensional sparse vector and there are only a few nonzero elements . $\mathrm{N}(\mathrm{L})$ is a $\mathrm{M} \times 1$ dimensional noise vector. Formula 1 can also be described as:

$$
\min \|\boldsymbol{X}\|_{0} \text { s.t. } \boldsymbol{Y}=\boldsymbol{G} \boldsymbol{X}+\boldsymbol{N}
$$

Formula 2 is a NP-hard problem. Therefore, the widely used methods are base pursuit represented by BP and greedy algorithm represented by OMP.

\subsection{Sparse Representation of Matched Field Source Localization}

Suppose that the area to be searched is two-dimensional region. As is shown in figure 1, the black dots on the left represent the receiving array and the right is the search area. The search area is divided into some grid points represented by white circles. The gray circle represents the position of the sound source. Because the target signal is sparse inspatial domain, we can use spatial mesh partition to realize the sparse representation of the acoustic target signal. Correspondence between signal and space is available after sparse representation which helps the acoustic localization.

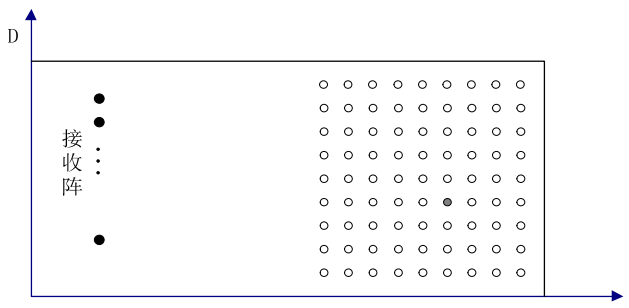

Figure 1. Sketch map about the search area discretization of acoustic source localization.

Based on the analysis of the above model, the matched field localization problem can be expressed as the following sparse model. The area of interest is dispersed into Q grid points, recorded as $\{\mathrm{r} 1$, $\mathrm{r} 2, \ldots \mathrm{rQ}\}$. Each element in the $\mathrm{X}$ indicates the pulse intensity of the signal at the grid point. Assume that there are $\mathrm{K}$ acoustic sources in the search area and $\mathrm{M}$ array elements to receive datum. Array received data [4] at $\mathrm{L}$ moment can be expressed as:

$$
y(l)=\boldsymbol{G} x(l)+n(l), l=1,2, \ldots, L
$$

$\mathrm{G}=[\mathrm{g}(\omega 0, \mathrm{r} 1) \mathrm{g}(\omega 0, \mathrm{r} 2) \ldots \mathrm{g}(\omega 0, \mathrm{rq})]$, which is called observation matrix.

$\mathrm{g}(\omega 0, \mathrm{rq})=[\mathrm{g} 1(\omega 0, \mathrm{rq}) \mathrm{g} 2(\omega 0, \mathrm{rq}) \ldots \mathrm{gM}(\omega 0, \mathrm{rq})] \mathrm{T}, \mathrm{q}=1,2, \ldots \mathrm{Q} . \mathrm{Q}$ represents the number of grids divided by the search space. Normally, Q is not less than K. M represents the number of elements. gi $(\omega 0, r q),(i=1,2, \ldots M)$ represents the Green function from the position rq to the array element numbered i. Each rq corresponds to a possible acoustic source location. L is the number of snapshots. If there is no acoustic source, the signal pulse is $0 . n(1)$ represents the signal noise at $\mathrm{L}$ moment.

\subsection{Model based on Compressed Sensing and Solution Using Joint Reconstruction}

Based on the compressed sensing theory, the matched field acoustic source localization problem can be expressed as:

$$
\boldsymbol{y}(l)=\boldsymbol{G} \boldsymbol{x}(l)+\boldsymbol{n}(l), l=1,2, \ldots, L
$$

$\mathrm{G}$ is a $\mathrm{M} * \mathrm{~N}$ dimensional measurement matrix. $\mathrm{Y}(\mathrm{L})$ is the sampled data after compression sampling. $Y(L)$ is a $M * 1$ dimensional vector. Because $M$ is less than $N, Y(L)$ is much smaller than the traditional array output value. Therefore, it reduces the requirement for the hardware and the amount of data processing. In order to ensure the accuracy of the estimate, we need multiple samplings. Then the formula 4 can be expressed as:

$$
\boldsymbol{Y}=\boldsymbol{G} \boldsymbol{X}+\boldsymbol{N}
$$

$\mathrm{Y}=[\mathrm{y}(1), \mathrm{y}(2), \ldots \mathrm{y}(\mathrm{L})], \mathrm{X}=[\mathrm{x}(1), \mathrm{x}(2), \ldots \mathrm{x}(1)], \mathrm{N}=[\mathrm{n}(1), \mathrm{n}(2), \ldots \mathrm{n}(\mathrm{L})] . \mathrm{L}$ is the number of snapshots. The solution of formula 5 can be optimized as:

$$
\min \|\boldsymbol{X}\|_{0} \text { s.t. } \boldsymbol{Y}=\boldsymbol{G} \boldsymbol{X}+\boldsymbol{N}
$$

This procedure requires the use of joint reconstruction algorithms, such as SOMP, M-FOCUSS, convex relaxation mixed norm algorithm. To solve formula 6 , this paper uses the convex relaxation hybrid norm algorithm. While $\varepsilon$ equals 0 , formula 6 can be expressed as:

$$
\min \|\boldsymbol{x}(l)\|_{l_{0}} \text { s.t. } \boldsymbol{y}(l)=\boldsymbol{G} \boldsymbol{x}(l)+\boldsymbol{n}(l)
$$


To solve equation 7 , we can obtain the location of the non-zero elements in signal $x(1)$. Then, according to the position of non-zero elements in each $\mathrm{x}(1)$ we can know the location of the source [5].To sum up, the acoustic localization based on compressed sensing can be summarized as:

Disperse the location of interest into Q grid points. Arrange every grid point in order. We assume that acoustic targets just locate on these discrete grid points. Then, the position of the acoustic source will correspond to the grid position in the space of interest.

2. According to the formula 1, obtain the spatial sparse representation model of the array received signal. Select the measurement matrix and do compressive sampling to obtain the array output data, which is just the formula 3.

3. Use the joint reconstruction algorithm to solve formula 7. Finally, obtain the location of the acoustic source by finding the position of the nonzero elements in $\mathrm{x}(1)$.

\subsection{Simulation Results Analysis}

Based on the above theory, the feasibility and effectiveness of the proposed method are verified by experiment simulation. Marine environment is assumed that the velocity of sound in sea water is $1500 \mathrm{~m} / \mathrm{s}$ and the sea water density is $1.0 \mathrm{~g} / \mathrm{cm} 3$. Sound speed in sea bottom is $1800 \mathrm{~m} / \mathrm{s}$ and the density of sea bed is $1.8 \mathrm{~g} / \mathrm{cm} 3$. We choose uniform linear array (ULA) . There are 7 elements in the array. The number of possible location is $\mathrm{Q}$ which is equal to 5000. In addition, the scanning step is one. The horizontal scanning range is between 0 and 100 meters, while the vertical one is between 0 and 50 meters. We add white Gaussian noise to the single and the input SNR is 10dB.The actual sound source position coordinates are $(40,25)$ and $(80,25)$.

The Feasibility of the Method

Under the above experimental conditions, the simulation experiment is carried out and the simulation results are shown in figure 2 . It can be seen from the figure that the proposed method can accurately position the target in the situation of few snapshots. As a result, we can conclude that the proposed method is feasible.

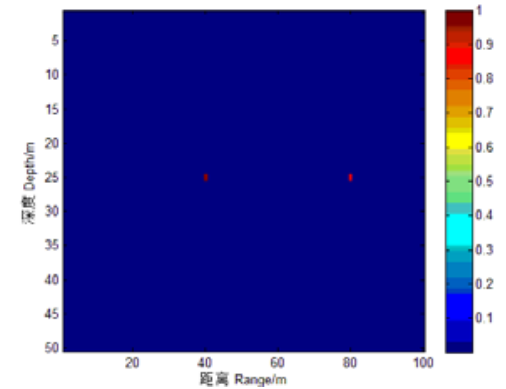

Figure 2. The positioning results achieved by the method this paper proposed.

\subsubsection{Single Snapshot Location}

When there is only one snapshot in time domain, MVDR matched field algorithm is ineffective. As a result, we only compare the localization performance of Bartlett algorithm and joint reconstruction algorithm. The simulation results are shown in figure 3 and figure 4 . Figure 3 is the result of the joint reconstruction algorithm whose positioning results are $(40,25)$ and $(80,25)$. This method can accurately locate the underwater acoustic. Bartlett positioning result is shown in figure 4 . It can be seen that only one position of the source can be clearly located. Worse still, the position is slightly biased and has a high side lobe. Therefore, the positioning effect is not ideal.

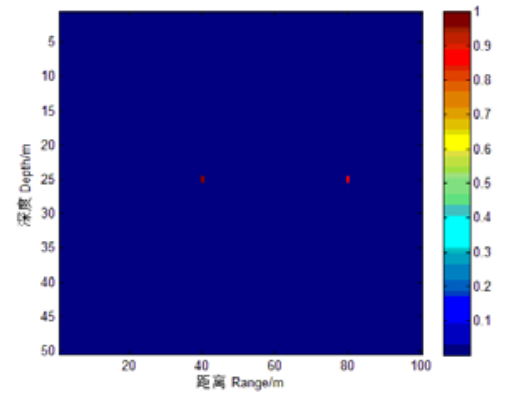

Figure 3. Matched field source localization based on joint reconstruction algorithm 


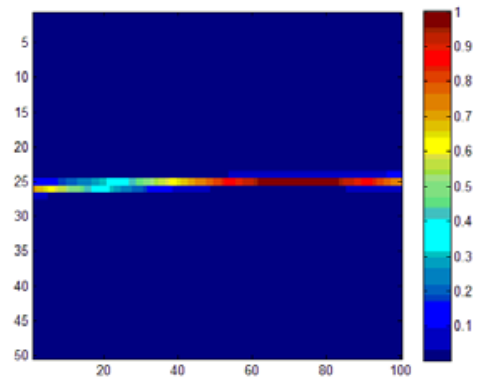

Figure 4. Matched field source localization based on Bartlett algorithm

\subsubsection{Multiple Snapshots Location}

Compare the positioning performance of joint reconstruction algorithm, Bartlett and MVDR algorithm. Results are shown in follow three figures. It can be seen from the figures that traditional matched field localization methods have larger side lobe, while the matching field positioning based on compressed sensing can obtain higher accuracy.

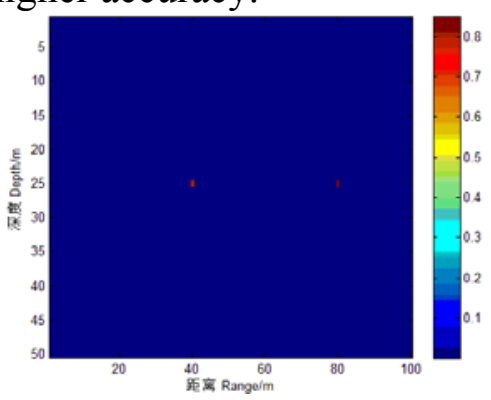

Figure 5. Matched field source localization based on joint reconstruction algorithm

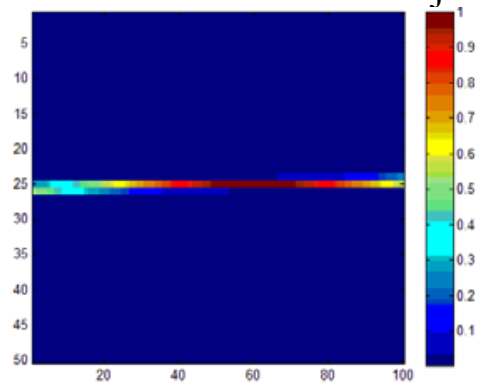

Figure 6. Matched field source localization based on Bartlett algorithm

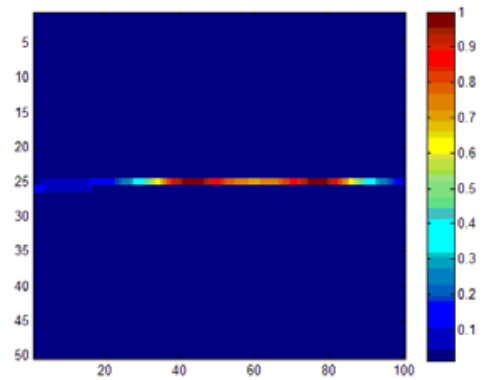

Figure 7. Matched field source localization based on MVDR algorithm

\section{Conclusion}

In this paper, a new method about matched field source localization based on compressed sensing is proposed. This method can effectively solve the problem that conventional matched field localization method has high side lobe. The simulation results show that the method could increase the DOA estimation accuracy of underwater acoustic target at less array elements and less snapshots which means saving the cost of positioning. A new effective way is proposed to solve the problem of matched field acoustic localization. 


\section{References}

[1]. Wang Xuezhi, Tu Ying, Wu Ketong, et al. (2012)Single vector hydrophone's 3D source location by applying matched field processing[J]. Technical Acoustics, 01:72-76.

[2]. Zhang Yu, Sun Dajun, Shi Junjie, et al. (2014) Passive location realization of single vector hydrophone by applying matched field[J]. Transducer and Microsystem Technologies, 04:21-23.

[3]. Wang Qi, Wang Ying-min, Zhu Guolei. (2014) Matched Field Processing Based on feature Extraction [J].Fire Control \& Command Control, 05:97-100+106.

[4]. You Wei, He Zishu, Hu Jinfeng. (2013)Skywave Radar Altitude Estimation Algorithm Based on Matched-field Processing [J]. Journal of Electronics \& Information Technology, 02:401-405.

[5]. Xiang Long-feng, Sun Chao. (2014) An estimation method of ship radiated noise level based on matched field processing [J]. ACTA ACOUSTICA, 05:570-576. 